\title{
The Possible Role of the Tibial Plateau Angle for the Severity of Osteoarthritis in Dogs with Cranial Cruciate Ligament Rupture
}

\author{
Yukihiro FUJITA ${ }^{1)}$, Yasushi HARA ${ }^{1}$, Hiroki OCHI ${ }^{1)}$, Yoshinori NEZU1), Yasuji HARADA ${ }^{1)}$, Takuya YOGO ${ }^{1)}$, \\ Hiromitsu ORIMA $^{2)}$ and Masahiro TAGAWA ${ }^{1)}$ \\ ${ }^{1)}$ Divisions of Veterinary Surgery and ${ }^{2)}$ Veterinary Radiology, Department of Veterinary Science, Faculty of Veterinary Medicine, Nippon \\ Veterinary and Life Science University 1-7-1 Kyonan-cho, Musashino-shi, Tokyo 180-8602, Japan
}

(Received 5 July 2005/Accepted 7 March 2006)

ABSTRACT. The purpose of this study was to determine factors correlated with the severity of radiographic osteoarthritis (OA) scoring in dogs with cranial cruciate ligament rupture (CrCLR). Three radiographs of stifle joints (craniocaudal, mediolateral, and mediolateral radiograph with 90 degree flexion of the stifle and tarsal joints) were obtained from 36 dogs with CrCLR (Clinical group) and from 22 dogs without stifle joint disease (Control group). Information about these dogs was collected from the owners and from medical records. Radiographic OA scores in each dog in the clinical group were determined from radiographs using a numeric grading system previously reported. The tibial plateau angle (TPA) in each dog in both groups was measured on mediolateral radiographs with 90 degree flexion of the stifle and tarsal joints. The Mann-Whitney's $U$ test was used for comparing variables between the clinical group and the control group, and Spearman's rank correlation test was used for evaluating correlations between radiographic OA scores and variables in the clinical group. No significant differences were detected between the clinical group and the control group for any of the variables. There were two positive correlations; one between the radiographic OA score and TPA $(r=0.395, p=0.014)$; and the other between body weight and OA score $(r=0.399, \mathrm{p}=0.013)$ in the clinical group. Our results indicate that body weight and TPA could affect the severity of the radiographic OA score in dogs with CrCLR.

KEY WORDS: canine, cranial cruciate ligament rupture, osteoarthritis, radiographic score, tibial plateau angle.

Cranial cruciate ligament rupture (CrCLR) is one of the most common injuries in dogs, and the major cause of osteoarthritis (OA) or degenerative joint disease in the stifle joint [19]. The cause of rupture is often unknown, and the optimal mode of treatment remains controversial [8].

Cranial tibial thrust (CrTT), defined as a cranially directed force generated by tibial compression, is responsible for cranial drawer motion in the cranial cruciate ligament (CrCL) deficient stifle, and is opposed by the CrCL [15-17]. Previous reports showed that there is a close relationship between the tibial plateau angle (TPA) and the amount of CrTT generated during axial tibial loading [14, 20]. The proximal tibias exhibited cranial bowing, resulting in abnormally high TPAs, and have been associated with CrCLR. They also reported that this deformity with high TPA apparently alters the biomechanics of the stifle joint and leads to degenerative joint disease and CrCLR [12]. Despite the similar report [9], several other groups have reported controversial results $[13,21]$. One study using Labrador Retrievers with (42 dogs) and without CrCLR (39 dogs) reported that mean TPAs were 23.5 degrees and 23.6 degrees respectively; an insignificant difference [13]. Another study concluded that many dogs with a high TPA do not develop CrCLR, although TPA may be associated with damage to the CrCL [21].

To our knowledge, there are no references in the veterinary literature that describe the relationship between TPA and the severity of osteoarthritis (OA) in dogs with CrCLR. We hypothesized that OA severity of dogs with a higher TPA is more progressive than that of dogs with a lower
TPA. The purpose of this study was to determine whether there is a relationship between the magnitude of TPA and radiographic OA severity and, as in previous studies, whether dogs with CrCLR had a greater TPA than dogs of similar breed, age, weight and lameness duration without CrCL disease.

\section{MATERIALS AND METHODS}

Dogs: Thirty six dogs of 13 breeds (10 males, 5 neutered males, 11 females, 10 spayed females) presented to the animal medical center at Nippon Veterinary and Life Science University (NVLU) with CrCLR (Clinical group). The most commonly represented breeds were Labrador Retriever (25\%), Golden Retriever (11\%) and Siberian Husky (11\%). CrCLR was present in both joints in three dogs. All joints with CrCLR showed positive drawer sign. Other joint instabilities were not detected. CrCLR was confirmed in 37 of 40 joints by exploring the stifle joints during surgery. Duration of lameness of the affected limb was obtained from the owners. Twenty two dogs of 10 breeds without stifle disease ( 8 males, 3 neutered males, 9 females, 2 spayed females) presented to the animal medical center at NVLU were used as control (Control group). Lack of signs of OA or CrCLR was confirmed by orthopedic and radiographic examinations. In dogs in both groups, two mediolateral radiographs (mediolateral, and mediolateral radiograph with 90 degree flexion of the stifle and tarsal joints) for both stifle joints, and one craniocaudal radiograph were taken for measuring TPA and evaluating radio- 


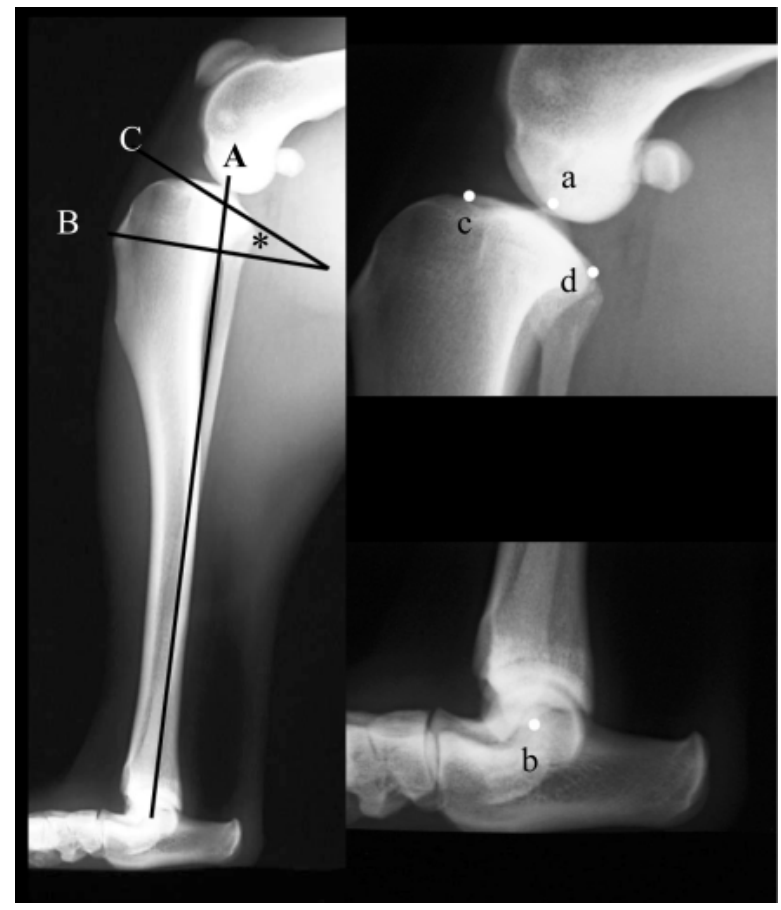

Fig. 1. Measurement of tibial plateau angle (TPA). The long axis of the tibia (line "A") was determined by the midpoint between the tibial intercondyler eminences (white dot "a") and the center of the talus (white dot "b"). A line was drawn perpendicular to the long axis of the tibia (line "B"). The medial tibial plateau (line "C") was determined by its most cranial (white dot "c") and most caudal margin (white dot "d"). The angle between a line "B" and a line "C" was defined as the TPA $\left({ }^{*}\right)$.

graphic scores.

Measurement of TPA: TPA was measured for all joints according to the method by Warzee C. et al. [20] as shown in Fig. 1. Each mediolateral radiograph with 90 degree flexion of the stifle and tarsal joints was used for measuring TPA. Briefly, the tibia is positioned parallel to the radiographic film. Both the femoral and tibial condyles are perfectly superimposed, and the mediolateral radiograph also includes the tibiotarsal joint. On each its radiograph, the long axis of the tibia was determined by the midpoint between the tibial intercondyler eminences and the center of the talus. A line was drawn perpendicular to the long axis of the tibia. The medial tibial plateau was determined by its most cranial and most caudal margin. The angle between a line perpendicular to the tibial axis and the tibial plateau was defined as the TPA. TPAs were obtained from 40 stifle joints in the clinical group and 41 stifle joints in the control group. The TPA was determined as the mean of three measurements for each stifle.

Radiographic grading system: Severity of OA in stifle joints of dogs in the clinical group was graded on radiographs according to a scoring system established previously [4]. This system ranged from 0 (no OA sign: osteophytes absent) to 4 (severe: osteophytes present on patella, femoral trochlear groove, medial and lateral femoral condyles, and tibial plateau; subchondral scerosis of fermoral condyles) (Fig. 2). Radiographic scores were obtained from 40 stifle joints in the clinical group.

Statistical analyses: Significant differences for each measurement between the clinical group and the control group were evaluated by using the Mann-Whitney's $U$ test. In the clinical group, relationships between the radiographic OA score and variables (age, body weight, lameness duration and TPA) were determined by use of Spearman's rank correlation. For all comparisons, $\mathrm{p}<0.05$ was considered statistically significant.

\section{RESULTS}

Information on the clinical aspects of dogs in both the clinical and control groups is summarized in Table 1. Mean ages of dogs in the clinical group and the control group were 76.7 months and 37.3 months, and mean body weights were $26.4 \mathrm{~kg}$ and $27.7 \mathrm{~kg}$, respectively. In the clinical group, mean lameness duration was 18.1 weeks and mean radiographic score was 2.6 (range: $0-4$ ). Mean TPAs in the clinical group and the control group were 25.3 degrees, and 23.9 degrees, respectively. There were no significant differences in these variables between the two groups in this study.

Correlations between radiographic OA scores and the variables measured were determined, and the results are summarized in Table 2. Two positive correlations were noted; one between the radiographic OA score and TPA (r: $0.395, \mathrm{p}=0.0137$ ) and the other between the OA score and body weight (r: 0.399, $\mathrm{p}=0.0127$ ).

\section{DISCUSSION}

Stifle joint stability is maintained by both active and passive forces. Active forces are provided by the quadriceps and the long digital extensor as the extensor moments; and the hamstring muscles as the flexor moments are responsive to the activity of the dog. On the other hand, passive forces are primarily controlled by the CrCL, and secondarily by the medial meniscus [16]. Previous reports have stated that the $\mathrm{CrCL}$ is the only structure that prevents cranial drawer motion of the tibia $[1,10,17,18]$.

The present study assessed the relationship between radiographic scores and different variables. Our study detected two positive relationships; one between radiographic OA score and the magnitude of TPA, and the other between the radiographic OA score and body weight. Our results also showed that the degree of TPA in dogs with CrCLR was not significantly greater than that of dogs without CrCLR.

TPA might be considered as a factor in the pathogenesis for CrCLR in dogs $[1,16,17]$. CrTT is generated by loading body weight to stifle joint surface and TPA. Increases in posterior inclination of the tibial plateau have been associated with increases in cranial translation of the tibia during monopodial stance [2]. This finding could infer a similar 


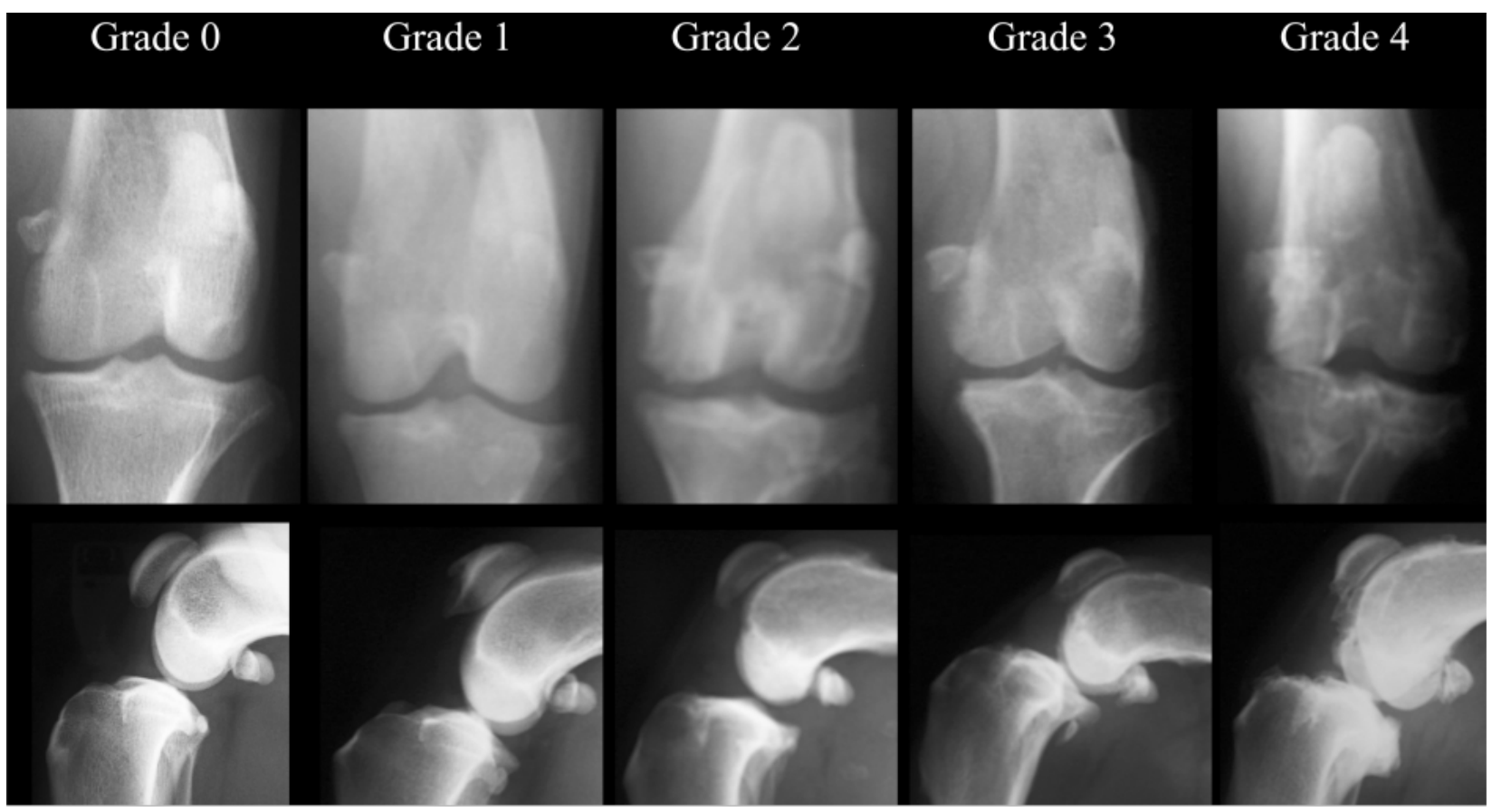

Fig. 2. Radiographic grading system. Radiographic grading was scored from craniocaudal and lateral radiographs. Grade 0 shows no osteoarthritic change (osteophytes absent) and Grade 4 shows severe osteoarthritic changes (osteophytes present on patella, femoral trochlear groove, medial and lateral femoral condyles, and tibial plateau; subchondral scerosis of fermoral condyles).

Table 1. Dogs in the clinical group and the control group

\begin{tabular}{|c|c|c|c|c|c|c|c|c|c|c|}
\hline & \multicolumn{6}{|c|}{ Clinical group } & \multicolumn{4}{|c|}{ Control group } \\
\hline & Mean & SD & Minimum & Median & Maximum & Mean & SD & Minimum & Median & Maximum \\
\hline Age (months) & 76.7 & 31.4 & 22.0 & 81.0 & 175.0 & 37.3 & 34.6 & 5.0 & 15.0 & 111.0 \\
\hline Body weight (kg) & 26.4 & 11.5 & 10.4 & 26.9 & 77.0 & 27.7 & 6.2 & 18.4 & 25.8 & 41.0 \\
\hline Lameness duration (weeks) & 18.1 & 24.8 & 1.0 & 11.0 & 96.0 & - & - & - & - & - \\
\hline Radiographic score $(0-4)$ & 2.6 & 1.3 & 0 & 3 & 4 & - & - & - & - & - \\
\hline Tibial plateau angle (degree) & 25.3 & 3.4 & 19.7 & 25.3 & 36.3 & 23.9 & 3.5 & 17.0 & 24.0 & 32.3 \\
\hline
\end{tabular}

Table 2. Correlations between radiographic score and variables

\begin{tabular}{llrl}
\hline & Variables & $\mathrm{r}$ & \multicolumn{1}{c}{$\mathrm{p}$} \\
\hline Radiographic score vs & Age & -0.314 & 0.0502 \\
& Body weight & 0.399 & 0.0127 \\
& Lameness duration & 0.186 & 0.2589 \\
& TPA & 0.395 & $0.0137 *$ \\
\hline
\end{tabular}

* Spearman's rank correlation $(\mathrm{p}<0.05)$.

situation in canine stifle joints. When a stifle joint in a dog with CrCLR has a greater posterior inclination of the tibial plateau, cranial translation of the tibia might increase. In in vitro experimental studies using canine cadavers, the tibial plateau leveling procedure was consistently successful in eliminating cranial translation of the tibia during axial loading $[14,20]$. This finding means that CrTT could be changed with the degree of TPA, and a greater CrTT means clinically that there is greater instability in the stifle joint.
CrTT can be confirmed by the tibial compression test [7]. A significant positive correlation between the magnitude of TPA and radiographic OA score was detected in the clinical group. This result may explain why the magnitude of TPA is one of the causes of OA progression following stifle instability with CrCLR. The greater TPA could increase stifle joint instability, and therefore $\mathrm{CrCL}$ deficient dogs with a higher TPA might exhibit a more progressive process than dogs with a lower TPA.

Generally, there are several causes of OA progression following CrCLR, such as acute or chronic disease process, body weight, lameness duration $[10,18]$. This study evaluated the relationship between the radiographic OA score and lameness duration. No significant correlation was detected between these variables, which could be due to the fact that dogs in the clinical group had various activity levels and we could not identify acute or chronic rupture of the CrCL from the limited information. In addition, one report showed the presence of OA in the stifle joint does not significantly cor- 
relate with clinical function evaluated by force platform gait analysis [6]. It is conceivable that our results could also be explained by this finding.

The present study also evaluated the relationship between radiographic OA score and body weight. Although there is clear evidence that being overweight is a significant risk factor for the development and progression of OA in human hands, hips and knees [5], the mechanism is still unclear. Excessive body weight might increase loading on weightbearing joints, and cyclic stresses could contribute to the degradation of articular cartilage and remodeling subchondral bone. Additionally, there is one report that body weight was a risk factor for CrCLR [3]. This study reported that the body weight of dogs with CrCLR was significantly greater than that of control dogs. Another report also showed that most dogs with cranial cruciate disease are of greater than ideal body condition [8]. A positive correlation between radiographic OA score and body weight in this study could be explained by increasing stress on stifle joint due to excessive body weight.

Previously, several investigators reported on the role of TPA for CrCLR in dogs. The one study reported that dogs with CrCLR had a significantly greater TPA than dogs without CrCL disease (mean TPA $23.8^{\circ}$ and $18.1^{\circ}$, respectively) [9]. Another group reported that there was no significant difference in the magnitude of TPA between dogs with CrCLR and dogs without CrCLR (mean TPA $23.5^{\circ}$ and $23.6^{\circ}$, respectively) [13]. According to these reports, the conclusion of whether or not TPA is one of the causes for CrCLR is still controversial. Previous report also showed that the mean TPA of Greyhounds without disease was $22.5^{\circ}$ and the authors concluded that this degree of TPA from Greyhounds could be considered normal for dogs [21]. In our study, the mean TPA of dogs with CrCLR was $25.3^{\circ}$ and that of dogs without CrCLR was $23.9^{\circ}$. The steepest TPA was found in the clinical group $\left(36.3^{\circ}\right)$ and the smallest TPA was found in the control group $\left(17.0^{\circ}\right)$. Even though there is a tendency for dogs with CrCLR to have a greater TPA, there is no significant difference in the present study. It was impossible to evaluate whether or not there is a significant difference between the TPA of the clinical group in our study and Greyhounds previously reported as normal. Significant difference between these TPAs might exist. In addition, further controlled studies are necessary to conclude the role of TPA for CrCLR in a large population, since breed peculiarities might exist $[9,13,21]$.

According to the results of our study the magnitude of TPA and body weight might be risk factors for the progression of and the degree of radiographic OA severity in dogs with CrCLR. However, further studies should be performed that include evaluation of the relationships between TPA or radiographic OA scoring and other disease related factors, such as the type or severity of meniscal injury, degree of pain, or clinical function. In addition, study of the longitudinal evaluation of the progression of $\mathrm{OA}$ is necessary to assess the role of TPA in OA progression in CrCL deficient stifles.

\section{REFERENCES}

1. Dejardin, L. M. 2003. Tibial plateau leveling osteotomy. pp. 2133-2143. In: Textbook of Small Animal Surgery, 3rd ed. (Slatter, D. ed.), Saunders, Philadelphia.

2. Dejour, H. and Bonnin, M. 1994. Tibial translation after anterior cruciate ligament rupture. J. Bone Joint Surg. 76-B: 745749.

3. Duval, J. M., Budsberg, S. C., Flo, G. L. and Sammarco, J. L. 1999. Breed, sex, and body weight as risk factors for rupture of the cranial cruciate ligament in young dogs. J. Am. Vet. Med. Assoc. 215: 811-814.

4. Elkins, A. D., Pechman, R., Kearney, M. T. and Herron, M. 1991. A retrospective study evaluating the degree of degenerative joint disease in the stifle joint of dogs following surgical repair of anterior cruciate ligament rupture. J. Am. Anim. Hosp. Assoc. 27: 533-540.

5. Felson, D. T. and Chaisoon, C. E. 1997. Understanding the relationship between body weight and osteoarthritis. Baillieres Clin. Rheumatol. 4: 671-681.

6. Gordon, W. J., Conzemius, M. G., Riedesel, E., Besancon, M. F., Evans, R., Wilke, V. and Ritter, M. J. 2003. The relationship between limb function and radiographic osteoarthritis in dogs with stifle osteoarthritis. Vet. Surg. 32: 451-454.

7. Henderson, R. A. and Milton, J. L. 1978. The tibial compression mechanism: A diagnostic aid in stifle injuries. $J$. Am. Anim. Hosp. Assoc. 14: 474-479.

8. Lampman, T. J., Lund, E. M. and Lipowitz, A. J. 2003. Cranial cruciate disease: Current status of diagnosis, surgery, and risk for disease. Vet. Comp. Orthop. Traumatol. 16: 122-126.

9. Morris, E. and Lipowitz, A. J. 2001. Comparison of tibial plateau angles in dogs with and without cranial cruciate ligament injuries. J. Am. Vet. Med. Assoc. 218: 363-366.

10. Piermattei, D. L. and Flo, G. L. 1997. The stifle joint. pp. 516579. In: Brinker, Piermattei, and Flo's Handbook of Small Animal Orthopedics and Fracture Repair, 3rd ed. (Permattei, D. L. and Flo, G. L. eds.), Saunders, Philadelphia.

11. Rayward, R. M., Thomson, D. G., Davies, J. V., Innes, J. F. and Whitelock, R. G. 2004. Progression of osteoarthritis following TPLO surgery: A prospective radiographic study of 40 dogs. J. Small Anim. Pract. 45: 92-97.

12. Read, R. A. and Robins, G. M. 1982. Deformity of the proximal tibia in dogs. Vet. Rec. 111: 295-298.

13. Reif, U. and Probst, C. 2003. Comparison of tibial plateau angles in normal and cranial cruciate deficient stifles of Labrador retrievers. Vet. Surg. 32: 385-389.

14. Reif, U., Hulse, D. A. and Hauptman, J. G. 2002. Effect of tibial plateau leveling on stability of the canine cranial cruciatedeficient stifle joint: An in vitro study. Vet. Surg. 31: 147-154.

15. Slocum, B. and Devine, T. 1983. Cranial tibial thrust: A primary force in canine stifle. J. Am. Vet. Med. Assoc. 184: 456459.

16. Slocum, B. and Slocum, T. D. 1993 Tibial plateau leveling osteotomy for repair of cranial cruciate ligament rupture in the canine. pp. 777-795. In: Veterinary Clinics of North America-Small Animal (Roush, J. K. ed.), Saunders, Philadelphia.

17. Slocum, B. and Slocum, T. D. 1998. Tibial plateau leveling osteotomy for cranial cruciate ligament rupture. pp. 12091215. In: Current Techniques in Small Animal Surgery, 4th ed. (Bojrab, M. J. ed.), Lea \& Febiger, Philadelphia.

18. Vasseur, P. B. Stifle joint. 2003. pp. 2090-2132. In: Textbook of Small Animal Surgery, 3rd ed. (Slatter, D. ed.), Saunders, Philadelphia. 
19. Vasseur, P.B. and Berry, C.R. 1992. Progression of stifle osteoarthrosis following reconstruction of the cranial cruciate ligament in 21 dogs. J. Am. Anim. Hosp. Assoc. 28: 129-136.

20. Warzee, C. C., Dejardin, L. M., Arnoczky, S. P. and Perry, R. L. 2001. Effect of tibial plateau leveling on cranial and caudal tibial thrust in canine cranial cruciate-deficient stifles: An in vitro experimental study. Vet. Surg. 30: 278-286.

21. Wilke, V. L., Conzemius, M. G., Besancon, M. F., Evans, R. B. and Ritter, M. 2002. Comparison of tibial plateau angle between clinically normal Greyhounds and Labrador retrievers with and without rupture of the cranial cruciate ligament. $J$. Am. Vet. Med. Assoc. 221: 1426-1429. 